onymen Geburten. Ich habe (allerdings vor fast 20 Jahren) einen Fachartikel über die anonymen Geburten im Journalclub der kinder- und jugendpsychiatrischen Universitätsklinik Basel vorgestellt. Es ging um eine Untersuchung von Frauen, welche die Möglichkeit der anonymen Geburt in Frankreich wählten. Es waren fast alles Frauen, die die Schwangerschaft monatelang verleugneten und sich erst eingestanden, schwanger zu sein, als ein Schwangerschaftsabbruch nicht mehr in Frage kam. Es ist auch bekannt, dass es ab und zu vorkommt, dass nicht nur Teenager, sondern auch ihr ganzes Umfeld eine Schwangerschaft verleugnen, sodass beispielsweise weder Mutter noch Turnlehrerin merken, wenn ein Mädchen immer runder wird.

Es wäre interessant zu wissen, wie das mit den Frauen aussieht, die ihr Kind in ein Babyfenster legen, ob sie auch ihre Schwangerschaft so lange verleugneten und das Kind vielleicht sogar ohne medizinische Hilfe zur Welt brachten. Da diese Frauen anonym bleiben, dürfte es allerdings schwierig sein, das zu erforschen.

Mir scheint das der entscheidende Punkt bei der Frage, ob Babyfenster ethisch akzeptabel sind: Wenn sie von Müttern benützt werden, die, überrumpelt durch eine vielleicht lange verdrängte Schwangerschaft, keinen anderen Ausweg sehen, als das Kind anonym abzugeben, dann ist das Babyfenster die bessere Alternative dazu, das Kind irgendwo auszusetzen oder gar zu töten oder an Vernachlässigung sterben zu lassen. In diesem Fall finde ich Babyfenster ethisch gerechtfertigt, weil sie zwar die Gefahr in sich bergen, dass das Kind nie erfährt, wer seine Mutter ist, aber immerhin dem Kind die Chance geben, am Leben zu bleiben. Wenn aber Frauen das Babyfenster wählen, die psychisch und vom sozialen Umfeld her auch imstande wären, das Kind normal zur Adoption freizugeben, dann ist das Babyfenster für das Kind die schlechtere Lösung, weil der Name der Mutter nicht registriert werden kann.

Mir scheint es einfach wichtig, nicht zu vergessen, dass gerade in unserem System, das viele Möglichkeiten bietet, mit einer ungewollten Schwangerschaft umzugehen (vom Schwangerschaftsabbruch über Unterstützung bei der Mutterschaft bis zur Freigabe zur Adoption), die Wahrscheinlichkeit recht gross ist, dass Frauen, die ihr Kind ins Babyfenster legen, sich zumindest subjektiv in einer sehr grossen Notlage befinden, da sie sonst andere Wege wählen würden. Oder aber dass es Frauen mit deutlichen psychischen Auffälligkeiten sind, die ihre Schwan- gerschaft sehr lange verdrängen und darum auch nicht überlegen, was mit dem Kind geschehen soll. Mir persönlich wäre die Möglichkeit einer anonymen Geburt im Spital lieber, da die Gebärende dort medizinisch betreut wäre und es auch Zeit gäbe, mit ihr zu überlegen, ob sie das Kind nicht auf dem normalen Weg zur Adoption geben will, sodass es später einmal ihren Namen erfahren kann.

$$
\text { Dr. med. Monika Diethelm, Uzwil }
$$

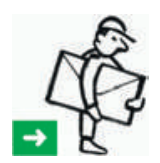

\section{Es geht primär um Leben}

Eine Frau, die ihr Neugeborenes in ein Babyfenster legt hat kurz zuvor geboren. Nach einer vielleicht mühevollen Schwangerschaft, wahrscheinlich einsam und alleingelassen, eine schmerzvolle und belastende Geburt, ganz allein ohne jegliche Hilfe durchgelitten, die Nachgeburt erwartet und abgenabelt, ihr Kind in den Armen, und jetzt die Frage: Wie weiter?

Vielleicht benötigt man um 500 miterlebte und betreute Geburten, im Spital und zu Hause, wie in meinem Arztsein, um zu erahnen, wie Gebären ohne Hebamme und ohne Beistand erfahrener Umgebung sein kann. Hier ist eigentliche Total-Mitmenschlichkeit gefordert. Wenigstens nachdenken, erwägen, versuchen, die Not einer solchen Mutter etwas nachzuvollziehen, ist wohl kaum zu viel verlangt.

Das Babyfenster als reale Hoffnung für das unmittelbare Überleben und Wohlergehen des noch hilflosen Neugeborenen. Es ist dieser Mutter in Not, in ihrer konkreten Situation, wohl kaum eine andere bessere Möglichkeit erreichbar anzubieten. Ihr Grundproblem ist doch: Sie ist allein. Allein gelassen, allein ver-lassen. Das Grundrecht des Kindes ist zu überleben. Dies hat sie ihm während der Schwangerschaft (vielleicht) gegen viele Widerstände erwahrt oder sogar erkämpft. Hier geht es ums Überleben, nicht um Identifizierbarkeit der Mutter. Diese wird ein ganzes volles Jahr durch das «Babyfenster» ermöglicht und empfohlen. Nur die betroffene Mutter weiss, ob die konkrete Situation es anrät und es sinnvoll macht, ihre Identität zu lüften. Wünsche und Rechte des Kindes dies betreffend, haben Zeit.

Von Babyfenstern nur deshalb abzuraten aus fiktiven «ethischen» Postulaten punkto
Identifikation ist wohl ein Nichterfassen der Realität. Es geht primär um Leben, um gutes, gesundes Überleben. Alles andere ist «Zugabe».

Dr. med. Josef Bättig, Muttenz

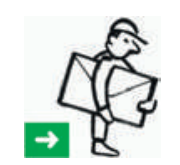

\section{Recht auf Kenntnis der Abstammung}

Lieber Herr Martin

Mit Interesse lese ich Ihre Kommentare zu den verschiedenen, meist aktuellen Themen unserer Zeit. Der Artikel über die Babyfenster [1] hat in mir tief prägende Erinnerungen ausgelöst an meine Zusammenarbeit mit einem der renommiertesten Kinderpsychiater in Zürich, meinem Lehrer und Begleiter in der Zeit der Assistenz in Pädiatrie. Erinnerungen auch an Jugendliche und junge Frauen, oft nach ihrer ersten oder zweiten Geburt, die sich als Adoptivkinder auf die intensive Suche nach ihrer Mutter, nach ihrer Herkunft machten. Ich erlebte die Not der Suche und der Verzweiflung wie auch die schwierige Akzeptanz der Tatsachen. Und da höre ich die Stimme des Kinderpsychiaters, die sich mit Vehemenz gegen die Adoption wehrte ... wie würde er sich wohl heute mit Vehemenz gegen die Babyfenster wehren. Denn seine Einstellung für die Offenlegung der Herkunft war geprägt von den gemachten Erfahrungen, wie ich sie später auch in der Praxis selber erleben musste. Auch bei einer Adoption hat das Kind ein Recht auf Kenntnis seiner Abstammung. Sollten nicht die Rahmenbedingungen für eine in Not stehende Frau in einer so reichen Gesellschaft, wie wir sie haben, derart gestaltet sein, dass weder Adoption noch Babyfenster nötig werden?

Ich danke Ihnen für Ihre kritische Haltung.

Hans-Ulrich Schär, Degersheim

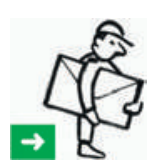

\section{Babyfenster und Ethik}

Die klare Stellungnahme von Jean Martin [1] zur ethischen Problematik um «Babyfenster» hat mich gefreut und entspricht meiner eigenen Haltung zu diesen offensichtlich immer 
populärer werdenden Einrichtungen. Dabei werden stets nur Notlage und Bedürfnis der Mutter berücksichtigt, nicht aber Bedürfnis und Recht des Kindes. Während in anderen Situationen (heterologe Insemination, Zwangsadoption) das Recht jedes Kindes, seine Abstammung zu kennen, öffentlich und gesetzlich gefordert wird, soll dies bei in «Babyfenstern» ausgesetzten Kindern nicht gelten. Es stehen genügend Hilfsmöglichkeiten für schwangere Frauen in Not zur Verfügung, «Babyfenster» sind ethisch fragwürdig und meines Erachtens nicht zu fördern.

Dr. med. Walter Felix Jungi, Wittenbach

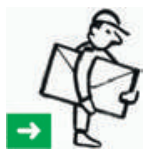

\section{Falsche Frage}

Die Alternative zum Babyfenster hiesse gemäss Herrn Martin Freigabe zur Adoption
[1]. Die Babyfenster sind aber aus der Bewegung der Abtreibungsgegner entstanden und sind aus meiner Sicht auch eine echte Alternative zu Abtreibung. Keine Mutter tut diese Schritte leicht, und niemand findet Babyfenster oder Abtreibung eine tolle Sache. Aber ich bin der festen Überzeugung, dass so einigen humanen Zellen mit diploidem Chromosomensatz es so doch ermöglicht wurde, zu einem Menschen heranzureifen. Niemand sieht in Babyfenstern ein Zeichen liberalen Fortschritts, wie von Herrn Martin erwähnt.

Wie steht es um das Recht des Kindes auf Wissen um seine Abstammung? Ich kenne diese Problematik sehr wohl, stamme ich doch aus einer Familie, in der man, um den Ruf der Familie zu wahren, die Mutter des unehelichen Kindes zwang, das Kind zur Adoption freizugeben. Ein Recht hat eigentlich nur einen Sinn, wenn es auch durchgeführt werden kann. Weiss man doch aus der Transplantationsmedizin, dass die Konstellation nicht so selten ist, dass der vermeintliche Er- zeuger des Erkrankten nicht der richtige Vater sein kann, was wohlweislich unter Verschluss gehalten wird. Die Argumentation mit Recht auf Kenntnis seiner Abstammung führt ad absurdum, weil die Realität komplexer ist und die Umsetzung in gewissen Fällen entweder nicht möglich oder auch gar nicht so hilfreich ist.

Am schwächsten finde ich die Argumentation der Gefahr des Drucks auf die junge Mutter. Hier kann ich einfach nur noch zynisch werden und sagen: Wie sieht es denn da aus, wenn sich jemand entschliesst, aktive Sterbehilfe zu beanspruchen oder sich für eine Abtreibung entscheidet? Es ist doch heuchlerisch, ein solches Argument hier einzubringen, aber den Einfluss der Umwelt in anderen ethischen Fragen zu ignorieren. Interessant. Wie sieht denn Ihr Weltbild aus, Herr Martin?

Dr. med. Markus Karl Stockmeyer, Basel 